\title{
Short telomere length and its correlation with gene mutations in myelodysplastic syndrome
}

Sang Mee Hwang ${ }^{1,2}$, Seon Young Kim³ ${ }^{3}$ Jung Ah Kim², Hee-Sue Park², Si Nae Park ${ }^{4}$ Kyongok $\mathrm{Im}^{4}$, Kwantae Kim², Sung-Min Kim ${ }^{4}$ and Dong Soon Lee $2,4^{*}$

\begin{abstract}
Background: Telomere erosion can lead to genomic instability and cancer progression. It has been suggested that the shortest telomere, not the average telomere length $(\mathrm{TL})$, is critical for cell viability. Some studies have shown shorter TL in myelodysplastic syndrome (MDS) patients but the critically short telomeres, the variability of TL within individual patient has not been evaluated. Thus, we aimed to investigate the TL of MDS patients and assessed the association of TL with recurrent genetic mutations in MDS.

Methods: We measured the TL of bone marrow nucleated cells for diagnostic samples at a single-cell level by quantitative fluorescence in situ hybridization (Q-FISH) for 58 MDS patients and analyzed the minimum, median, average, standard deviation, average of the 0th to 10th percentile TL within a patient, and the proportion of cells with $T L$ that is shorter than the lowest 10th percentile of the normal control (NC). The correlations of TL to clinical parameters, cytogenetic results, and genetic mutations were assessed.

Results: MDS patients showed eroded telomeres and narrow distribution compared to the $N C(P<0.001, P=0.018$, respectively). Patients with mutation showed significantly lesser cells with short $T L$, below the lowest 10th percentile of the NC $(P=0.017)$, but no differences in TL were found according to mutations/cytogenetic abnormalities except for CSF3R mutation. However, those patients with a high percentage ( $\geq 80 \%)$ of cells with short TL showed poorer overall survival $(P=0.021)$, and this was an independent prognostic factor, along with TP53, U2AF1 mutation, and high BM blast count $(P=0.044,0.001,0.004,0.012$, respectively).

Conclusions: The shortest $\mathrm{TL}$, which determines the fate of the cell, was significantly shorter, and higher burden of cells with short TL were found in MDS, which correlated with poor survival, suggesting the need to measure TL in single cells by Q-FISH.
\end{abstract}

Keywords: Telomere length, Quantitative fluorescence in situ hybridization, Single cell, Mutation

\section{Background}

Telomeres are non-coding, repetitive sequences of DNA at the ends of the chromosomes of eukaryotic cells which become shorter as cells divide, and when telomere attrition reaches its limit, cell proliferation arrest, senescence, and apoptosis can occur [1,2]. Shortened telomeres can cause end-to-end fusion of chromosomes, which results in

\footnotetext{
* Correspondence: soonlee@snu.ac.kr

${ }^{2}$ Department of Laboratory Medicine, Seoul National University College of Medicine, Seoul, Republic of Korea

${ }^{4}$ Cancer Research Institute, Seoul National University College of Medicine,

Seoul, Republic of Korea

Full list of author information is available at the end of the article
}

genomic instability and contributes to carcinogenesis [3]. Thus, telomeres and their maintenance have been studied in many hematologic malignancies [4-6].

Myelodysplastic syndromes (MDS) are clonal hematopoietic stem cell diseases characterized by cytopenia, dysplasia, and ineffective hematopoiesis, with an increased risk of transformation to acute myeloid leukemia (AML) $[7,8]$. Due to its predisposition to AML, genomic instability has been studied as the key to the pathogenesis of MDS, and thus, many studies have assessed the telomere components of MDS [9-11]. MDS patients have shown a shorter average telomere length (TL) compared 
to normal control (NC), but the association of TL to other clinical parameters in patients with MDS has been inconsistent $[9,11,12]$.

Many somatic mutations have been identified in patients with MDS, involving various pathways including epigenetic regulation, RNA splicing machinery, and transcription factors [13-15], which help elucidate the pathophysiology of the disease. A recent study showed that telomere dysfunction causes aberrant RNA splicing by repressing the splicing gene expression [16]. However, the correlation of TL with various genetic mutations other than telomerase complex genes has not been thoroughly explored $[17,18]$.

Moreover, most previous studies have used Southern blot analysis, quantitative PCR, or flow fluorescence in situ hybridization (FISH) for the analysis of TL $[9,11,19,20]$. However, these studies only assessed the mean or median $\mathrm{TL}$ of the patient whereas it is believed that the cell with the shortest telomere is actually the critical factor leading telomere dysfunction [3, 21]. Performing quantitative-FISH (Q-FISH) for telomeres allows the measurement of TL at a single-cell level with the measurement of the distribution of TL of within individuals as well as the shortest TL [22]. Telomeres of individual chromosomes are stained with peptic nucleic acid probes for telomeres, and the fluorescence intensity of the telomeric signal is measured with fluorescence-based automatic microscope [23]. The fluorescence intensity is normalized by the use of an additional centromeric probe, and the telomere to centromere $(\mathrm{T} / \mathrm{C})$ fluorescence intensity ratio is considered to be the surrogate of TL since the fluorescence intensity of the telomeric signal is known to be proportional to the TL [24]. Inclusion of the centromeric probe also allows the selection of single cells in which two centromeric signals are observed and the normalization of the signal for hybridization [23]. Performing Q-FISH in interphase nuclei further allows measurement of each value in numerous nucleated cells. Therefore, we measured the TL of bone marrow (BM) nucleated cells of patients with MDS at diagnosis by Q-FISH, evaluated the correlation of the TL with genetic mutations and clinical parameters, and explored the usefulness of TL parameters as a prognostic marker.

\section{Methods}

\section{Patients}

This study examined 58 patients diagnosed with MDS (37 men and 21 women) at Seoul National University Hospital between January 2004 and December 2014. This study was approved by the institutional review board of Seoul National University Hospital (1604-082-754). The samples included in this study were from diagnostic samples of MDS. The characteristics of the MDS patients are shown in Table 1. BM aspirates from 23 individuals who underwent marrow biopsy and showed no evidence of hematologic malignancy were included as the NC. The bone marrow of the NC showed no evidence of bone marrow involvement and showed normal karyotype.

\section{Karyotyping-Giemsa banding}

A standard protocol was used to perform Giemsa banding with heparinized whole blood marrow samples [25]. A minimum of 20 metaphase cells were karyotyped according to the International System for Human Cytogenetic Nomenclature (ISCN) [26, 27].

\section{Interphase fluorescence in situ hybridization for MDS} Interphase FISH tests (del(5q)/-5, del(7q)/-7, trisomy 8 , $\operatorname{del}(20 \mathrm{q})$, trisomy $1 / 1 \mathrm{q}+$ ) were performed. The probes used were Vysis LSI EGR1 (5q31)/D5S23, D5S721 Dual color probe kit, Vysis LSI D7S522 (7q31)/CEP7 FISH probe, Vysis CEP8(Dz82) Spectrum Green Probe, Vysis D20S108 FISH probe, and Vysis LSI 1p36/LSI1q25 and LSI 19q13/19p13 Dual color probe (Abbott Molecular, Downers Grove, IL, USA). These five types of FISH items were selected based on the previous report of recurrent cytogenetic changes in Korean patients with MDS [28]. FISH was performed with mononuclear cells of BM aspirates at the time of BM examination as described in a previous study [28].

\section{Telomere quantitative fluorescence in situ hybridization} Cryopreserved BM nucleated cells in fixative after FISH preparation were used for telomere analysis. Q-FISH was performed using a Cy3-labelled telomere PNA (peptide nucleic acid) FISH kit (DakoCytomation Denmark A/S, Glostrup, Denmark) and a FITC-labeled PNA probe for the centromere of chromosome 2 (kindly provided by DakoCytomation). One microliter of the probe for the centromere of chromosome 2 was added to $10 \mu \mathrm{L}$ of the telomere probe. Telomere and centromere Q-FISH hybridizations were performed according to the manufacturer's instructions. Interphase Q-FISH images were captured with a Zeiss Axioplan 2 imaging microscope (Carl Zeiss MicroImaging $\mathrm{GmbH}$, Munchen, Germany) equipped with ISIS software (MetaSystems GmbH, Altlussheim, Germany) (Fig. 1). For TL measurements, the ISISTelomere module (MetaSystems) was used as described previously [23]. The software calculates a $\mathrm{T} / \mathrm{C}$ fluorescence intensity ratio, which is a measure of $\mathrm{TL}$, for individual cells. At least 25 interphase nuclei were scanned for each patient to measure the TL. The cell with the shortest TL is represented as the minimum TL and the lower 25th percentile value of each individual is expressed as the "Q1" TL. The median, the average and the distribution of TL, standard distribution (SD), and the average of the 0th to 10th percentile TL for each individual was calculated from the total cells of which the TL was measured. Furthermore, the percentage of cells with TL 
Table 1 Characteristics of MDS patients $(n=58)$

\begin{tabular}{ll}
\hline Characteristics & Number (\%) \\
\hline Gender (Male/female) & $37: 21$ \\
Median age (year, range) & $69(18-86)$ \\
Age (years) & \\
$<60$ & $22(37.9)$ \\
$\geq 60$ & $36(62.1)$
\end{tabular}

Complete blood count at bone marrow sample collection

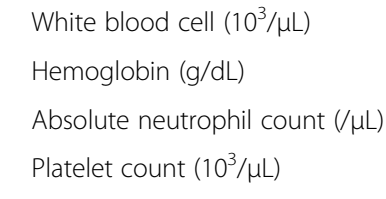

Median bone marrow blast (\%, range)

WHO category

$\begin{array}{ll}\text { RCUD } & 12(20.7) \\ \text { RARS } & 4(6.9) \\ \text { RCMD } & 13(22.4) \\ \text { RAEB } & 20(34.5) \\ \text { MDS, U } & 9(15.5)\end{array}$

IPSS risk groups

$\begin{array}{ll}\text { Low } & 8(13.8) \\ \text { Intermediate-1 } & 28(48.3) \\ \text { Intermediate-2 } & 15(25.9) \\ \text { High } & 7(12.1) \\ \text { Revised IPSS } & \\ \text { Very low } & 4(6.9) \\ \text { Low } & 14(24.1) \\ \text { Intermediate } & 14(24.1) \\ \text { High } & 14(24.1) \\ \text { Very High } & 12(20.7) \\ \text { Cytogenetic results } & \\ \text { Karyotype } & \\ \text { Abnormal karyotype } & 32(55.2) \\ \text { Complex karyotype (>3 abnormalities) } & 9(15.5) \\ \text { del(5q)/ -5 } & 6(10.5) \\ \text { del(7q)/ -7 } & 8(14.0) \\ \text { Trisomy } 8 & 8(14.0) \\ \text { Abnormal 17p } & 3(5.2) \\ \text { del(20q) } & 5(8.8) \\ \text { Fluorescence in situ hybridization } & \\ \text { Abnormal chromosome } 1^{\text {a }} & 5(11.1) \\ \text { Abnormal chromosome 5 } & \\ \text { b } & (17.4)\end{array}$

$2.6(0.6-17.2)$

$8.0(4.4-12.2)$

$1151(80-11,345)$

$74.5(3-1029)$

$2.3(0.0-18.1)$

$2(20.7)$

(6.9)

$3(22.4)$

(15.5)

8 (13.8)

$28(48.3)$

$(25.9)$

High

$4(6.9)$

(24.1)

$14(24.1)$

$12(20.7)$

Cytogenetic results

Karyotype 
TapeStation System (Agilent, Santa Clara, CA, USA). A total target length of the $259-\mathrm{kb}$ region using the pairedend 150-bp rapid-run sequencing mode was performed on an Illumina Hiseq 2500 platform (Illumina, San Diego, CA, USA). The sequencing data calling process is described in Additional file 1.

\section{Statistical analysis}

Categorization of MDS patients was performed according to the 2008 World Health Organization (WHO) classification of tumors of hematopoietic and lymphoid tissues [8], the International Prognostic Scoring System (IPSS), and the revised IPSS et al. [36, 37]. The correlation of TL with various clinical parameters, BM findings, cytogenetic abnormalities, and mutations found in multi-gene panels was assessed.

The gene mutations were evaluated separately and in subgroups according to the function of genes. Ten gene subgroups are splicing machinery, DNA methylation, chromatin modification, transcription factor, receptor/kinases, RAS pathway, cell signaling, DNA repair/cell cycle, cohesion, and miscellaneous. The genes in each subgroup are listed in the Additional file 1: Table S1. The $\chi 2$ test and Fisher's exact test were used to compare categorical variables. Pairwise correlations between TLs and gene mutations, cytogenetic results, and categorical clinical parameters were evaluated using Kendall tau-b $\left(T_{\mathrm{b}}\right)$ correlation. For linear parameters, Pearson's $r$ correlation was used. The comparisons of TLs between patients with and without a certain mutation were made with the Mann-Whitney method for genes that showed mutation in more than $5.0 \%$ of the patients.

The overall survival (OS) was calculated from the date of diagnosis to the date of death from any cause and compared by Kaplan-Meier method (log-rank test). Univariable and multivariable Cox analyses were performed to assess variables as prognostic factors for survival. Backward LR selection was performed for multivariable Cox analysis. Harrell's C-index was calculated for the proposed multivariable Cox model and the revised IPSS. Statistical analyses were performed using the SPSS version 22.0 (IBM Inc., Chicago, IL, USA) and $R$ statistical program (http://www.r-project.org). $P$ values $<0.05$ were considered to be statistically significant.

\section{Results}

Telomere lengths and clinical profiles

We compared the telomere lengths (TLs) of the MDS patients $(n=58)$ with those of the NC $(n=23)$, according to several parameters (Table 2). The clinical characteristics of the MDS patients included in this study are shown in Table 1 . There was no significant difference in age and sex ( $P=0.175,0.796$, respectively) between the MDS patients and NC. However, the number of cells assessed in the MDS patients was smaller $(P=0.004)$ than that assessed in the NC due to insufficient nuclei in the patient samples. All of the TL parameters were significantly lower in the MDS patients than the NC $(P<0.001$ for all the TL except $\mathrm{SD}, P=0.018$ for $\mathrm{SD})$. The distribution of the telomere length of each individual was narrower in the MDS patients than the NC $(P=0.018)$.

We also calculated the lowest 10th percentile TL value of the NC group and found out that $52.1( \pm 24.9) \%$ of MDS patients' cells were under this length (Table 2). These results show that not only the mean TL of the MDS patients is shorter than that of the NC but also a large proportion of MDS patients' cells are shorter than the lowest 10th percentile TL of the NC.

TL was compared for WHO categories and IPSS risk groups. The TL parameters were not different among the clinical subgroups (the average of the 0th-10th percentile TL and the median TL shown in Fig. 2, the other TL

Table 2 Telomere length parameters of MDS patients and normal control

\begin{tabular}{|c|c|c|c|}
\hline \multirow[t]{2}{*}{ Parameters } & \multicolumn{2}{|l|}{ Average $\pm S D$} & \multirow{2}{*}{$\begin{array}{l}P \\
\text { value }\end{array}$} \\
\hline & $\operatorname{MDS}(n=58)$ & $\mathrm{NC}(n=23)$ & \\
\hline Age & $63.7 \pm 14.6$ & $60.9 \pm 9.5$ & 0.175 \\
\hline Gender (M/F) & $37: 21$ & $16: 7$ & 0.796 \\
\hline No. of cells assessed for telomere lengths & $77.6 \pm 38.9$ & $98.1 \pm 26.3$ & 0.004 \\
\hline \multicolumn{4}{|l|}{ Telomere lengths ( $\mathrm{T} / \mathrm{C}$ ratio) } \\
\hline Minimum & $1.97 \pm 1.47$ & $4.41 \pm 1.36$ & $<0.001$ \\
\hline 25th percentile (Q1) & $5.49 \pm 3.04$ & $11.79 \pm 3.06$ & $<0.001$ \\
\hline Median & $8.56 \pm 4.45$ & $15.92 \pm 3.93$ & $<0.001$ \\
\hline Average & $10.08 \pm 4.73$ & $17.29 \pm 4.42$ & $<0.001$ \\
\hline Average of $0-10$ percentile & $2.95 \pm 1.76$ & $6.64 \pm 1.74$ & $<0.001$ \\
\hline Standard Deviation & $6.67 \pm 3.01$ & $8.07 \pm 2.73$ & 0.018 \\
\hline Cells under the 10th percentile of the NC (\%) & $52.1 \pm 24.9$ & & \\
\hline
\end{tabular}



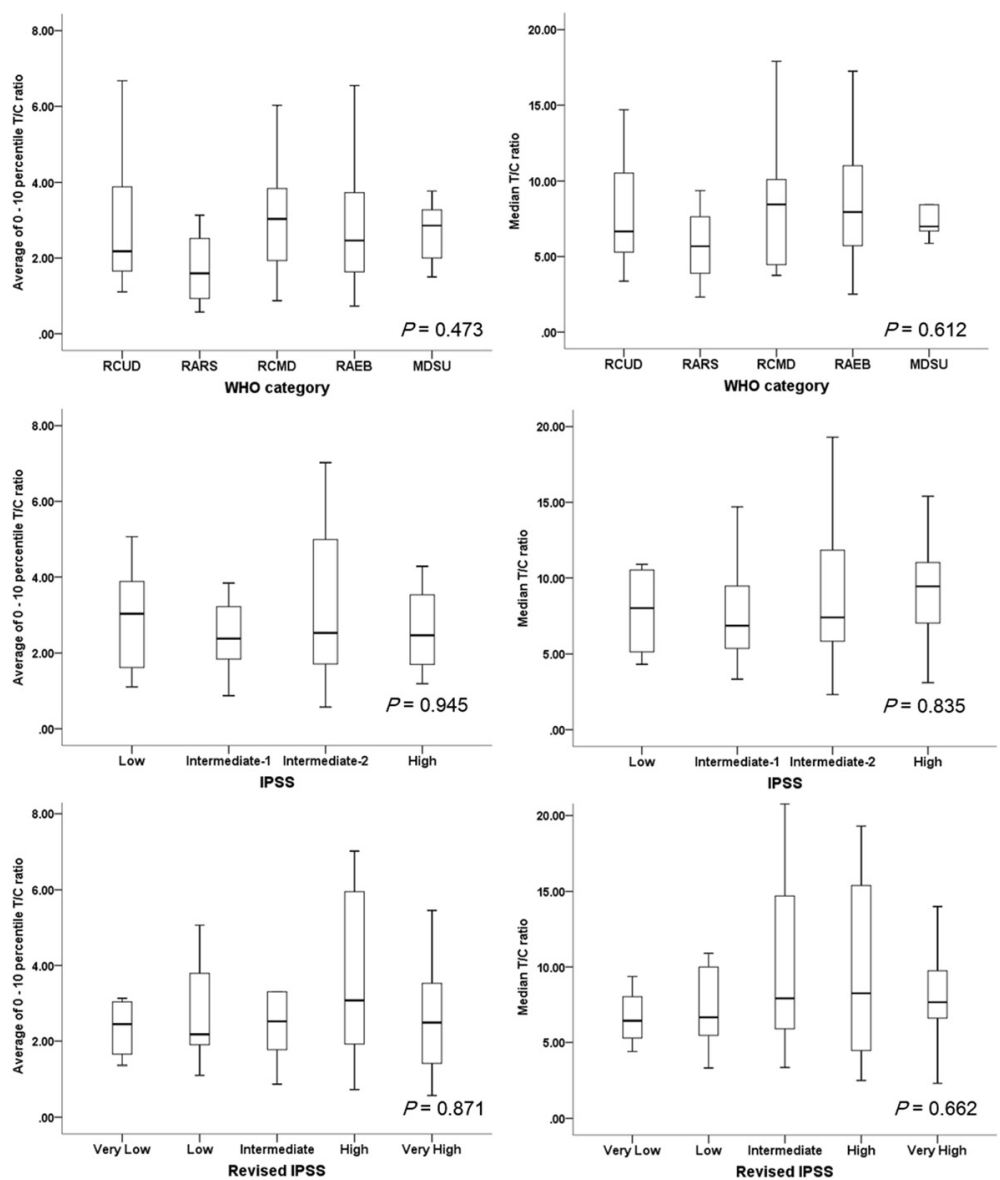

Fig. 2 Telomere lengths according to the World Health Organization (WHO) categories, International Prognostic Scoring System (IPSS), and revised IPSS subgroups. T/C telomere to centromere

parameters are in the Additional file 1: Table S2). Additional comparison of TL was made according to the complete blood count and BM findings. Patients with low hemoglobin $(<8 \mathrm{~g} / \mathrm{dL})$ showed a higher percentage of cells with TL less than the 10th percentile of normal control $(P=0.020)$. However, the other TL parameters were not significantly different in those patients with hemoglobin values less than vs. equal to/ greater than $8 \mathrm{~g} / \mathrm{dL}$. There were no significant differences in the TL according to absolute neutrophil count, platelet count, BM blast count, fibrosis, or cellularity (Additional file 1: Table S3).

\section{Multi-gene panel results}

Among the 87 genes, we found mutations of 43 genes in more than one patient. Forty-five patients $(77.6 \%)$ had showed mutation in one or more of the genes. Only the gene mutations found in more than $5 \%$ of the samples are shown in Fig. 3. Grouping the 87 genes by gene function, genes related to splicing were most frequently mutated $(34.5 \%)$, following the genes for transcription factors (31.0\%), chromatin modification (25.9\%), DNA methylation (19.0\%), receptor/kinases (15.5\%), and RAS pathways $(8.6 \%)$. The associations of gene mutations and/or cytogenetic results are shown in Fig. 4. Kendall tau-b $\left(T_{\mathrm{b}}\right)$ correlation coefficients greater than 0.40 were present between $S R S F 2$ and SETBP1 mutation $\left(T_{\mathrm{b}}=\right.$ $0.432, P=0.001), I D H 2$ and SRSF2 mutation $\left(T_{\mathrm{b}}=0.432\right.$, $P=0.001), T P 53$ mutation and $\operatorname{del}(5 \mathrm{q}) /-5\left(T_{\mathrm{b}}=0.520\right.$, $P<0.001)$, TP53 mutation and $\operatorname{del}(7 \mathrm{q}) /-7 \quad\left(T_{\mathrm{b}}=0.564, P<\right.$ $0.001)$, TP53 mutation and abnormalities of $17 \mathrm{p}\left(T_{\mathrm{b}}=0.583\right.$, $P<0.001)$, del $(5 \mathrm{q}) /-5$ and abnormality of $17 \mathrm{p}\left(T_{\mathrm{b}}=0.431\right.$, $P=0.001)$. Significant correlations were also present between DNMT3A vs. IDH2 mutation, DNMT3A vs. SETBP1 mutation $\left(T_{\mathrm{b}}=0.390, P=0.003\right.$, respectively $)$, U2AF1 mutation vs. trisomy $8\left(T_{\mathrm{b}}=0.379, P=0.005\right)$, $\operatorname{del}(7 \mathrm{q}) /-7$ vs. abnormal $17 \mathrm{p} \quad\left(T_{\mathrm{b}}=0.357, P=0.008\right)$, SF3B1 vs. TET 2 mutation $\left(T_{\mathrm{b}}=0.343, P=0.010\right), D N M T 3 A$ vs. TET2 mutation $\left(T_{\mathrm{b}}=0.263, P=0.047\right), I D H 2$ vs. $R U N X 1$ mutation, $R U N X 1$ vs. SETBP1 mutation, IDH 2 vs. SETBP1 mutation $\left(T_{\mathrm{b}}=0.297, P=0.025\right.$, respectively). Comparing the association between gene categories and cytogenetic abnormalities; DNA methylation vs. cell signaling-related genes $\left(T_{\mathrm{b}}=0.391, P=0.003\right)$, DNA methylation vs. DNA 


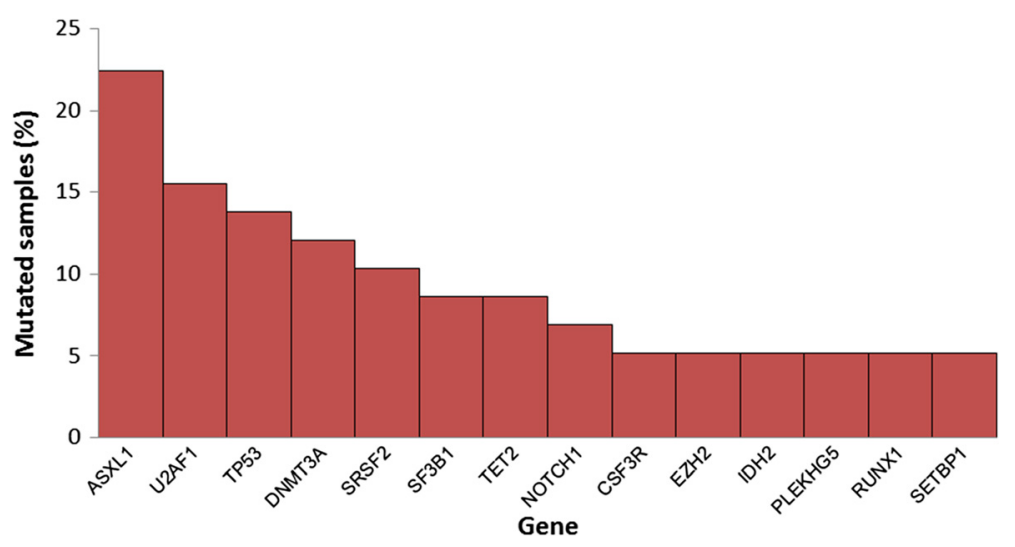

Fig. 3 The percentage of samples that was found with specific gene mutations in genes with mutations in more than $5 \%$ of the patients

repair/cell cycle-related genes $\left(T_{\mathrm{b}}=0.389, P=0.003\right)$, RAS pathway vs. splicing-related genes $\left(T_{\mathrm{b}}=0.294, P=0.026\right)$, splicing-related genes vs. trisomy $8\left(T_{\mathrm{b}}=0.338, P=0.011\right)$, transcription factor genes vs. $\operatorname{del}(5 \mathrm{q}) /-5\left(T_{\mathrm{b}}=0.276, P=\right.$ $0.039)$, transcription factor genes vs. $\operatorname{del}(7 \mathrm{q}) /-7\left(T_{\mathrm{b}}=0.399\right.$, $P=0.003)$, transcription factor genes vs. abnormality of $17 \mathrm{p}$ $\left(T_{\mathrm{b}}=0.362, P=0.007\right)$ were shown to have significant correlations (Additional file 1: Figure S1).

\section{Telomere length and genetic mutations/cytogenetic} abnormalities

We analyzed whether somatic mutations had a relationship with TL. Table 3 shows the TL of patients according to whether or not they have a certain somatic mutation.
Patients without gene mutations had shorter TL than those with mutation. All of the TL parameters showed significant differences (minimum, Q1, median, average, average of $0-10$ percentile, SD, percentage of cells under the 10th percentile of the NC) between those patients with or without gene mutations $(P=0.043,0.041,0.025$, $0.014,0.030,0.007,0.017$, respectively). Looking at specific mutations, the difference in TL was only present for those with or without CSF3R mutation. Patients without CSF3R mutation $(n=55)$ had a significantly higher percentage of cells whose length was below the lowest 10 th percentile of the NC than those with CSF3R mutation $(n=3)(P=$ 0.037). In addition, comparisons were made for the TL according to gene groups. However, there were no

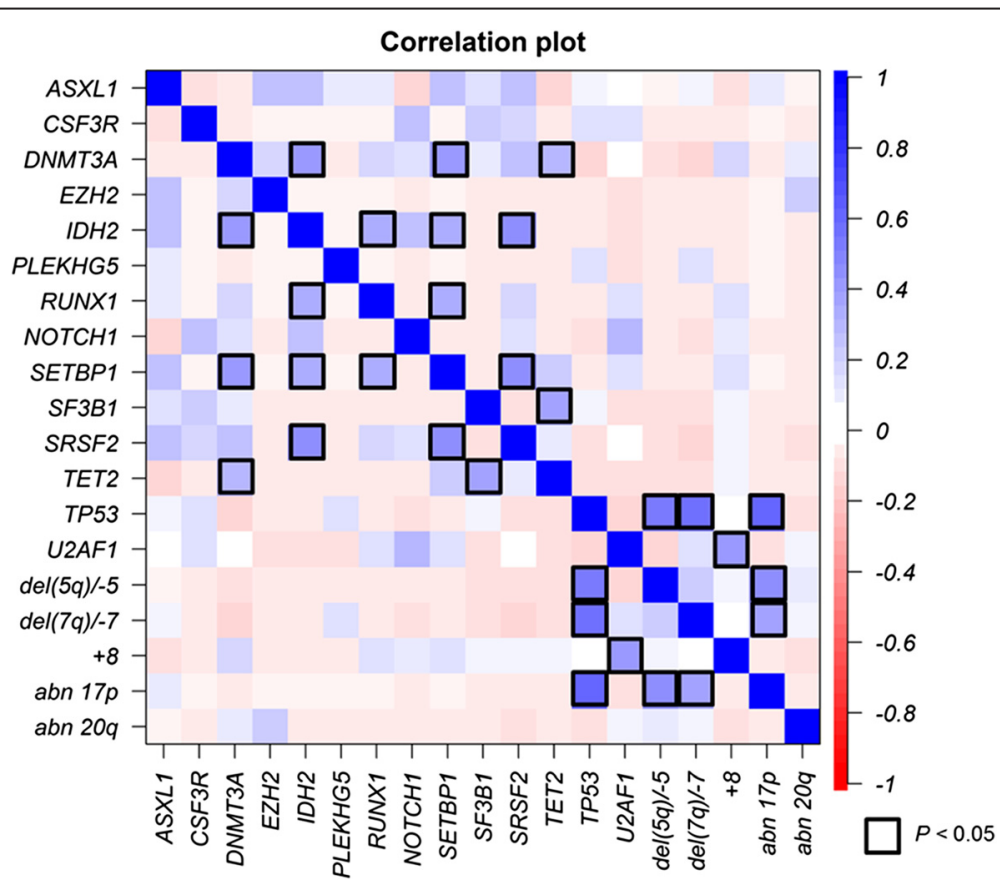

Fig. 4 Correlations between gene mutations and cytogenetic changes. Significant correlations are marked with bold border 
Table 3 Telomere lengths according to gene mutations or cytogenetic changes

\begin{tabular}{|c|c|c|c|c|c|c|c|c|c|c|c|c|c|c|c|c|c|c|}
\hline \multirow{2}{*}{$\begin{array}{l}\text { Gene or } \\
\text { cytogenetic } \\
\text { changes }\end{array}$} & & \multirow[t]{2}{*}{ Number } & \multirow[t]{2}{*}{ Mean age } & \multirow{2}{*}{$\begin{array}{l}P \\
\text { value }\end{array}$} & \multicolumn{14}{|c|}{ Telomere lengths ( $\mathrm{T} / \mathrm{C}$ ratio) } \\
\hline & & & & & Minimum & $P$ value & Q1 & $P$ value & Median & $P$ value & Average & $P$ value & $\begin{array}{l}\text { Average } \\
\text { of } 0-10 \\
\text { percentile }\end{array}$ & $P$ value & $\begin{array}{l}\text { Standard } \\
\text { deviation }\end{array}$ & $P$ value & $\begin{array}{l}\text { Cells under } \\
\text { the } 10 \text { th } \\
\text { percentile } \\
\text { of normal } \\
\text { control (\%) }\end{array}$ & $P$ value \\
\hline \multirow{2}{*}{$\begin{array}{l}\text { Any gene } \\
\text { mutation }\end{array}$} & Mut(+) & 45 & $65.4 \pm 12.2$ & 0.356 & $2.14 \pm 1.53$ & 0.043 & $5.82 \pm 3.07$ & 0.041 & $8.99 \pm 4.17$ & 0.025 & $10.68 \pm 4.56$ & 0.014 & $3.18 \pm 1.82$ & 0.030 & $7.12 \pm 2.97$ & 0.007 & $48.2 \pm 23.6$ & 0.017 \\
\hline & $\operatorname{Mut}(-)$ & 13 & $57.9 \pm 20.6$ & & $1.38 \pm 1.11$ & & $4.34 \pm 2.80$ & & $7.12 \pm 5.26$ & & $8.00 \pm 4.90$ & & $2.14 \pm 1.32$ & & $5.13 \pm 2.74$ & & $65.8 \pm 25.5$ & \\
\hline \multirow[t]{2}{*}{$A S X L 1$} & Mut(+) & 13 & $65.4 \pm 10.8$ & 0.837 & $2.13 \pm 2.29$ & 0.461 & $5.32 \pm 3.79$ & 0.557 & $8.04 \pm 4.83$ & 0.595 & $9.39 \pm 5.20$ & 0.450 & $2.97 \pm 2.28$ & 0.689 & $5.78 \pm 2.40$ & 0.310 & $57.4 \pm 24.4$ & 0.396 \\
\hline & Mut(-) & 45 & $63.2 \pm 15.7$ & & $1.92 \pm 1.17$ & & $5.54 \pm 2.85$ & & $8.72 \pm 4.39$ & & $10.28 \pm 4.63$ & & $2.94 \pm 1.62$ & & $6.93 \pm 3.15$ & & $50.6 \pm 25.1$ & \\
\hline \multirow[t]{2}{*}{ CSF3R } & $\operatorname{Mut}(+)$ & 3 & $57.3 \pm 21.1$ & 0.438 & $2.21 \pm 0.99$ & 0.438 & $7.87 \pm 2.87$ & 0.087 & $12.43 \pm 3.71$ & 0.062 & $14.23 \pm 4.18$ & 0.067 & $3.82 \pm 2.29$ & 0.459 & $9.23 \pm 2.43$ & 0.119 & $23.9 \pm 13.8$ & 0.037 \\
\hline & Mut(-) & 55 & $64.0 \pm 14.4$ & & $1.96 \pm 1.50$ & & $5.36 \pm 3.03$ & & $8.36 \pm 4.42$ & & $9.86 \pm 4.69$ & & $2.90 \pm 1.74$ & & $6.53 \pm 3.00$ & & $53.7 \pm 24.5$ & \\
\hline \multirow[t]{2}{*}{ DNMT3A } & $\operatorname{Mut}(+)$ & 7 & $72.0 \pm 8.9$ & 0.117 & $1.66 \pm 0.77$ & 1.000 & $4.38 \pm 1.25$ & 0.439 & $6.77 \pm 1.58$ & 0.425 & $8.14 \pm 1.66$ & 0.453 & $2.50 \pm 0.75$ & 0.907 & $5.43 \pm 1.88$ & 0.779 & $55.6 \pm 15.4$ & 0.312 \\
\hline & Mut(-) & 51 & $62.6 \pm 15.0$ & & $2.01 \pm 1.55$ & & $5.65 \pm 3.20$ & & $8.82 \pm 4.67$ & & $10.35 \pm 4.96$ & & $3.01 \pm 1.86$ & & $6.84 \pm 3.12$ & & $51.7 \pm 26.0$ & \\
\hline \multirow[t]{2}{*}{ EZH2 } & Mut(+) & 3 & $71.7 \pm 0.6$ & 0.240 & $3.95 \pm 3.95$ & 0.379 & $7.71 \pm 5.74$ & 0.547 & $10.66 \pm 6.28$ & 0.525 & $12.57 \pm 7.71$ & 0.502 & $4.44 \pm 3.43$ & 0.342 & $7.13 \pm 3.97$ & 0.869 & $62.3 \pm 12.5$ & 0.594 \\
\hline & Mut(-) & 55 & $63.3 \pm 14.9$ & & $1.86 \pm 1.22$ & & $5.37 \pm 2.88$ & & $8.45 \pm 4.39$ & & $9.95 \pm 4.59$ & & $2.87 \pm 1.65$ & & $6.65 \pm 3.00$ & & $51.6 \pm 25.4$ & \\
\hline \multirow[t]{2}{*}{ IDH2 } & $\operatorname{Mut}(+)$ & 3 & $66.7 \pm 9.1$ & 0.843 & $3.08 \pm 2.20$ & 0.232 & $6.18 \pm 4.53$ & 0.974 & $10.04 \pm 6.42$ & 0.715 & $11.70 \pm 5.50$ & 0.570 & $3.91 \pm 2.73$ & 0.525 & $8.05 \pm 0.99$ & 0.232 & $38.7 \pm 29.1$ & 0.525 \\
\hline & Mut(-) & 55 & $63.5 \pm 14.9$ & & $1.91 \pm 1.43$ & & $5.46 \pm 3.00$ & & $8.49 \pm 4.39$ & & $9.99 \pm 4.73$ & & $2.90 \pm 1.72$ & & $6.60 \pm 3.08$ & & $52.9 \pm 24.8$ & \\
\hline \multirow[t]{2}{*}{ NOTCH1 } & Mut(+) & 4 & $64.0 \pm 17.1$ & 0.824 & $2.47 \pm 0.84$ & 0.189 & $8.01 \pm 4.97$ & 0.348 & $11.94 \pm 7.12$ & 0.363 & $14.43 \pm 7.72$ & 0.236 & $4.24 \pm 2.29$ & 0.248 & $9.48 \pm 3.33$ & 0.067 & $35.3 \pm 31.7$ & 0.332 \\
\hline & Mut(-) & 54 & $63.7 \pm 14.6$ & & $1.93 \pm 1.51$ & & $5.31 \pm 2.84$ & & $8.32 \pm 4.19$ & & $9.76 \pm 4.38$ & & $2.85 \pm 1.71$ & & $6.46 \pm 2.92$ & & $53.4 \pm 24.2$ & \\
\hline \multirow[t]{2}{*}{ PLEKHG5 } & $\operatorname{Mut}(+)$ & 3 & $58.7 \pm 10.3$ & 0.324 & $1.01 \pm 0.52$ & 0.158 & $2.86 \pm 2.05$ & 0.111 & $5.07 \pm 2.45$ & 0.138 & $6.99 \pm 3.37$ & 0.291 & $1.57 \pm 1.17$ & 0.119 & $5.95 \pm 2.90$ & 0.843 & $70.3 \pm 18.0$ & 0.193 \\
\hline & Mut(-) & 55 & $64.0 \pm 14.9$ & & $2.02 \pm 1.49$ & & $5.64 \pm 3.04$ & & $8.76 \pm 4.47$ & & $10.25 \pm 4.76$ & & $3.02 \pm 1.77$ & & $6.71 \pm 3.04$ & & $51.1 \pm 25.0$ & \\
\hline \multirow[t]{2}{*}{ RUNX1 } & $\operatorname{Mut}(+)$ & 3 & $56.7 \pm 10.6$ & 0.193 & $2.13 \pm 1.27$ & 0.690 & $4.90 \pm 1.76$ & 0.843 & $10.16 \pm 5.47$ & 0.641 & $11.73 \pm 5.44$ & 0.570 & $2.83 \pm 0.92$ & 0.766 & $8.91 \pm 4.08$ & 0.193 & $58.0 \pm 14.9$ & 0.641 \\
\hline & Mut(-) & 55 & $64.1 \pm 14.8$ & & $1.96 \pm 1.49$ & & $5.53 \pm 3.11$ & & $8.48 \pm 4.44$ & & $9.99 \pm 4.73$ & & $2.95 \pm 1.80$ & & $6.55 \pm 2.95$ & & $51.8 \pm 25.4$ & \\
\hline \multirow[t]{2}{*}{ SETBP1 } & Mut(+) & 3 & $66.7 \pm 20.0$ & 0.895 & $1.76 \pm 0.32$ & 0.690 & $4.20 \pm 1.02$ & 0.547 & $6.48 \pm 1.80$ & 0.594 & $7.61 \pm 2.03$ & 0.459 & $2.41 \pm 0.43$ & 0.869 & $4.76 \pm 2.01$ & 0.246 & $58.6 \pm 18.7$ & 0.690 \\
\hline & Mut(-) & 55 & $63.5 \pm 14.5$ & & $1.98 \pm 1.51$ & & $5.56 \pm 3.11$ & & $8.68 \pm 4.54$ & & $10.22 \pm 4.81$ & & $2.98 \pm 1.81$ & & $6.78 \pm 3.04$ & & $51.8 \pm 25.3$ & \\
\hline \multirow[t]{2}{*}{$S F 3 B 1$} & Mut(+) & 5 & $63.6 \pm 11.2$ & 0.788 & $2.09 \pm 1.60$ & 0.872 & $5.13 \pm 1.68$ & 0.747 & $7.99 \pm 2.51$ & 0.851 & $9.87 \pm 3.23$ & 0.768 & $2.72 \pm 1.48$ & 0.893 & $7.79 \pm 3.99$ & 0.553 & $47.2 \pm 19.2$ & 0.517 \\
\hline & Mut(-) & 53 & $63.7 \pm 15.0$ & & $1.96 \pm 1.48$ & & $5.53 \pm 3.16$ & & $8.62 \pm 4.61$ & & $10.10 \pm 4.87$ & & $2.97 \pm 1.80$ & & $6.57 \pm 2.94$ & & $52.6 \pm 25.5$ & \\
\hline \multirow[t]{2}{*}{ SRSF2 } & Mut(+) & 6 & $73.8 \pm 8.0$ & 0.101 & $2.40 \pm 1.72$ & 0.446 & $6.47 \pm 3.76$ & 0.558 & $10.10 \pm 5.48$ & 0.446 & $11.42 \pm 5.68$ & 0.508 & $3.70 \pm 2.26$ & 0.374 & $6.89 \pm 2.55$ & 0.737 & $42.4 \pm 30.5$ & 0.416 \\
\hline & Mut(-) & 52 & $62.5 \pm 14.8$ & & $1.92 \pm 1.45$ & & $5.38 \pm 2.98$ & & $8.39 \pm 4.35$ & & $9.93 \pm 4.65$ & & $2.86 \pm 1.70$ & & $6.65 \pm 3.09$ & & $53.2 \pm 24.3$ & \\
\hline \multirow[t]{2}{*}{ TET2 } & Mut(+) & 5 & $75.8 \pm 6.1$ & 0.021 & $2.85 \pm 1.69$ & 0.131 & $7.06 \pm 2.70$ & 0.086 & $10.06 \pm 2.89$ & 0.162 & $12.11 \pm 3.22$ & 0.138 & $3.91 \pm 1.91$ & 0.154 & $8.19 \pm 3.81$ & 0.316 & $33.4 \pm 18.5$ & 0.076 \\
\hline & Mut(-) & 53 & $62.6 \pm 14.7$ & & $1.89 \pm 1.44$ & & $5.35 \pm 3.06$ & & $8.43 \pm 4.57$ & & $9.89 \pm 4.83$ & & $2.86 \pm 1.74$ & & $6.53 \pm 2.94$ & & $53.9 \pm 24.8$ & \\
\hline \multirow[t]{2}{*}{ TP53 } & $\operatorname{Mut}(+)$ & 8 & $69.0 \pm 8.9$ & 0.471 & $1.81 \pm 1.23$ & 0.748 & $5.12 \pm 3.06$ & 0.974 & $8.44 \pm 4.08$ & 0.748 & $10.25 \pm 4.36$ & 0.650 & $2.67 \pm 1.84$ & 0.471 & $7.62 \pm 3.05$ & 0.260 & $47.2 \pm 27.0$ & 0.471 \\
\hline & Mut(-) & 50 & $62.8 \pm 15.3$ & & $1.99 \pm 1.52$ & & $5.55 \pm 3.07$ & & $8.59 \pm 4.55$ & & $10.05 \pm 4.83$ & & $2.99 \pm 1.77$ & & $6.52 \pm 3.01$ & & $52.9 \pm 24.7$ & \\
\hline
\end{tabular}


Table 3 Telomere lengths according to gene mutations or cytogenetic changes (Continued)

\begin{tabular}{|c|c|c|c|c|c|c|c|c|c|c|c|c|c|c|c|c|c|c|}
\hline \multirow[t]{2}{*}{ U2AF1 } & Mut(+) & 9 & $59.4 \pm 12.7$ & 0.179 & $2.02 \pm 1.01$ & 0.408 & $6.62 \pm 3.49$ & 0.225 & $10.39 \pm 5.60$ & 0.269 & $12.08 \pm 6.35$ & 0.318 & $3.49 \pm 1.69$ & 0.201 & $7.77 \pm 4.21$ & 0.512 & $47.6 \pm 27.7$ & 0.614 \\
\hline & Mut(-) & 49 & $64.5 \pm 15.0$ & & $1.96 \pm 1.55$ & & $5.29 \pm 2.95$ & & $8.23 \pm 4.20$ & & $9.71 \pm 4.36$ & & $2.85 \pm 1.78$ & & $6.47 \pm 2.75$ & & $53.0 \pm 24.6$ & \\
\hline \multirow[t]{2}{*}{ Karyotype } & Normal & 25 & $61.2 \pm 15.1$ & 0.416 & $2.00 \pm 1.95$ & 0.237 & $5.32 \pm 3.44$ & 0.385 & $7.95 \pm 4.46$ & 0.274 & $9.36 \pm 4.93$ & 0.222 & $2.93 \pm 2.09$ & 0.459 & $6.08 \pm 3.01$ & 0.216 & $57.7 \pm 26.6$ & 0.123 \\
\hline & Abnormal & 32 & $65.3 \pm 14.3$ & & $1.92 \pm 1.02$ & & $5.64 \pm 2.80$ & & $9.00 \pm 4.53$ & & $10.53 \pm 4.61$ & & $2.94 \pm 1.53$ & & $7.00 \pm 2.96$ & & $48.3 \pm 23.3$ & \\
\hline \multirow[t]{2}{*}{$\operatorname{del}(5 q) /-5$} & $(+)$ & 6 & $68.7 \pm 13.3$ & 0.393 & $2.18 \pm 1.20$ & 0.500 & $6.71 \pm 3.56$ & 0.393 & $10.28 \pm 4.58$ & 0.233 & $12.33 \pm 4.40$ & 0.177 & $3.78 \pm 2.16$ & 0.352 & $8.39 \pm 2.36$ & 0.048 & $35.4 \pm 28.4$ & 0.089 \\
\hline & $(-)$ & 51 & $62.9 \pm 14.9$ & & $1.93 \pm 1.52$ & & $5.35 \pm 3.02$ & & $8.34 \pm 4.48$ & & $9.75 \pm 4.76$ & & $2.84 \pm 1.73$ & & $6.39 \pm 3.00$ & & $54.4 \pm 24.1$ & \\
\hline \multirow[t]{2}{*}{$\operatorname{del}(7 q) /-7$} & $(+)$ & 8 & $61.5 \pm 18.6$ & 0.866 & $2.13 \pm 1.05$ & 0.302 & $6.58 \pm 3.00$ & 0.111 & $10.44 \pm 5.45$ & 0.175 & $11.59 \pm 4.90$ & 0.207 & $3.25 \pm 1.51$ & 0.261 & $7.06 \pm 2.96$ & 0.449 & $39.8 \pm 24.2$ & 0.096 \\
\hline & $(-)$ & 49 & $63.8 \pm 14.2$ & & $1.93 \pm 1.55$ & & $5.32 \pm 3.08$ & & $8.23 \pm 4.30$ & & $9.76 \pm 4.73$ & & $2.89 \pm 1.83$ & & $6.52 \pm 3.02$ & & $54.5 \pm 24.80$ & \\
\hline \multirow[t]{2}{*}{ Trisomy 8} & $(+)$ & 8 & $54.4 \pm 20.5$ & 0.123 & $1.87 \pm 1.02$ & 0.778 & $6.35 \pm 3.84$ & 0.628 & $11.09 \pm 6.69$ & 0.261 & $12.35 \pm 6.63$ & 0.291 & $2.95 \pm 1.64$ & 0.813 & $8.29 \pm 4.26$ & 0.302 & $51.3 \pm 27.8$ & 0.919 \\
\hline & $(-)$ & 49 & $65.0 \pm 13.2$ & & $1.97 \pm 1.56$ & & $5.36 \pm 2.96$ & & $8.12 \pm 3.97$ & & $9.64 \pm 4.34$ & & $2.94 \pm 1.82$ & & $6.32 \pm 2.69$ & & $52.6 \pm 24.8$ & \\
\hline \multirow[t]{2}{*}{ Abn 17p } & $(+)$ & 3 & $72.3 \pm 10.1$ & 0.307 & $2.72 \pm 1.47$ & 0.324 & $6.05 \pm 3.84$ & 0.749 & $8.89 \pm 5.48$ & 0.906 & $10.34 \pm 5.00$ & 0.880 & $3.72 \pm 2.24$ & 0.599 & $6.25 \pm 1.63$ & 0.801 & $45.4 \pm 33.9$ & 0.623 \\
\hline & $(-)$ & 54 & $63.0 \pm 14.8$ & & $1.91 \pm 1.49$ & & $5.47 \pm 3.07$ & & $8.52 \pm 4.49$ & & $10.00 \pm 4.78$ & & $2.90 \pm 1.77$ & & $6.62 \pm 3.06$ & & $52.8 \pm 24.8$ & \\
\hline \multirow[t]{2}{*}{$\operatorname{del}(20 q)$} & $(+)$ & 5 & $68.0 \pm 10.7$ & 0.632 & $2.16 \pm 0.55$ & 0.186 & $5.56 \pm 1.41$ & 0.519 & $8.13 \pm 2.15$ & 0.795 & $9.70 \pm 2.27$ & 0.712 & $3.13 \pm 0.73$ & 0.262 & $6.12 \pm 1.28$ & 0.880 & $44.4 \pm 15.0$ & 0.327 \\
\hline & $(-)$ & 52 & $63.1 \pm 15.0$ & & $1.94 \pm 1.55$ & & $5.49 \pm 3.20$ & & $8.58 \pm 4.66$ & & $10.05 \pm 4.94$ & & $2.92 \pm 1.85$ & & $6.65 \pm 3.11$ & & $53.2 \pm 25.8$ & \\
\hline \multirow[t]{2}{*}{ FISH 1} & $(+)$ & 5 & $54.0 \pm 25.2$ & 0.428 & $1.61 \pm 1.04$ & 0.562 & $7.03 \pm 4.89$ & 0.586 & $11.77 \pm 7.84$ & 0.368 & $13.19 \pm 7.37$ & 0.349 & $2.92 \pm 2.09$ & 0.903 & $8.99 \pm 3.72$ & 0.128 & $40.4 \pm 29.4$ & 0.314 \\
\hline & $(-)$ & 40 & $63.0 \pm 13.9$ & & $1.89 \pm 1.18$ & & $5.49 \pm 3.20$ & & $8.28 \pm 3.98$ & & $9.71 \pm 4.24$ & & $2.86 \pm 1.64$ & & $6.34 \pm 2.72$ & & $51.9 \pm 25.4$ & \\
\hline \multirow[t]{2}{*}{ FISH 5} & $(+)$ & 8 & $67.3 \pm 9.8$ & 0.485 & $1.80 \pm 1.20$ & 0.765 & $5.60 \pm 3.63$ & 0.921 & $9.17 \pm 4.77$ & 0.559 & $10.97 \pm 4.87$ & 0.450 & $2.92 \pm 2.23$ & 0.579 & $7.86 \pm 2.91$ & 0.123 & $43.4 \pm 30.7$ & 0.338 \\
\hline & $(-)$ & 38 & $61.3 \pm 16.2$ & & $1.85 \pm 1.15$ & & $5.45 \pm 2.86$ & & $8.55 \pm 4.52$ & & $9.88 \pm 4.64$ & & $2.87 \pm 1.54$ & & $6.33 \pm 2.87$ & & $52.1 \pm 24.5$ & \\
\hline \multirow[t]{2}{*}{ FISH 7} & $(+)$ & 9 & $69.3 \pm 7.0$ & 0.160 & $1.94 \pm 1.08$ & 0.605 & $5.57 \pm 2.66$ & 0.478 & $8.54 \pm 3.65$ & 0.643 & $10.08 \pm 4.10$ & 0.723 & $2.92 \pm 1.63$ & 0.870 & $6.69 \pm 2.74$ & 0.786 & $44.4 \pm 15.04$ & 0.461 \\
\hline & $(-)$ & 37 & $60.6 \pm 16.4$ & & $1.82 \pm 1.18$ & & $5.46 \pm 3.06$ & & $8.68 \pm 4.75$ & & $10.07 \pm 4.83$ & & $2.87 \pm 1.67$ & & $6.58 \pm 2.98$ & & $53.2 \pm 25.8$ & \\
\hline \multirow[t]{2}{*}{ FISH 8} & $(+)$ & 7 & $59.6 \pm 15.5$ & 0.490 & $1.90 \pm 1.10$ & 0.858 & $5.77 \pm 3.74$ & 1.000 & $9.70 \pm 5.85$ & 0.697 & $11.43 \pm 6.59$ & 0.697 & $3.01 \pm 1.76$ & 0.881 & $7.90 \pm 4.44$ & 0.549 & $55.6 \pm 27.2$ & 0.549 \\
\hline & $(-)$ & 39 & $62.8 \pm 15.5$ & & $1.83 \pm 1.17$ & & $5.43 \pm 2.86$ & & $8.47 \pm 4.30$ & & $9.82 \pm 4.29$ & & $2.85 \pm 1.65$ & & $6.37 \pm 2.55$ & & $49.7 \pm 25.5$ & \\
\hline \multirow[t]{2}{*}{ FISH 20} & $(+)$ & 4 & $64.3 \pm 7.6$ & 0.985 & $2.37 \pm 1.03$ & 0.173 & $5.82 \pm 1.49$ & 0.417 & $8.38 \pm 2.22$ & 0.721 & $9.85 \pm 2.20$ & 0.721 & $3.35 \pm 1.06$ & 0.229 & $6.02 \pm 1.08$ & 0.895 & $44.0 \pm 15.3$ & 0.510 \\
\hline & $(-)$ & 42 & $62.0 \pm 15.8$ & & $1.78 \pm 1.17$ & & $5.39 \pm 3.10$ & & $8.60 \pm 4.74$ & & $10.00 \pm 4.89$ & & $2.79 \pm 1.70$ & & $6.62 \pm 3.06$ & & $52.0 \pm 26.7$ & \\
\hline
\end{tabular}


significant differences in TL parameters by 10 different gene groups (Additional file 1: Table S4). Since TL varies according to age in the normal population, the age was compared between the mutational status of the genes or gene groups. Significant differences were only found for TET2 mutational status and the presence/ absence of mutation in DNA methylation genes category. Patients with TET2 mutation and mutations of DNA methylation were older than those without mutation $(P=0.021,0.008$, respectively). The TL was not significantly different between patients with or without certain cytogenetic abnormalities by karyotyping or FISH
(Table 3). In summary, we found that the presence of any gene mutation, but not the cytogenetic results, was related to TL.

\section{Survival analysis}

The OS was compared among different clinical subgroups (Fig. 5). There were significant differences in survival among different IPSS and the revised IPSS risk groups $(P=0.010,0.004$, respectively). However, no difference in OS was observed among the WHO categories $(P=$ 0.462 ) by Kaplan-Meier survival analysis.
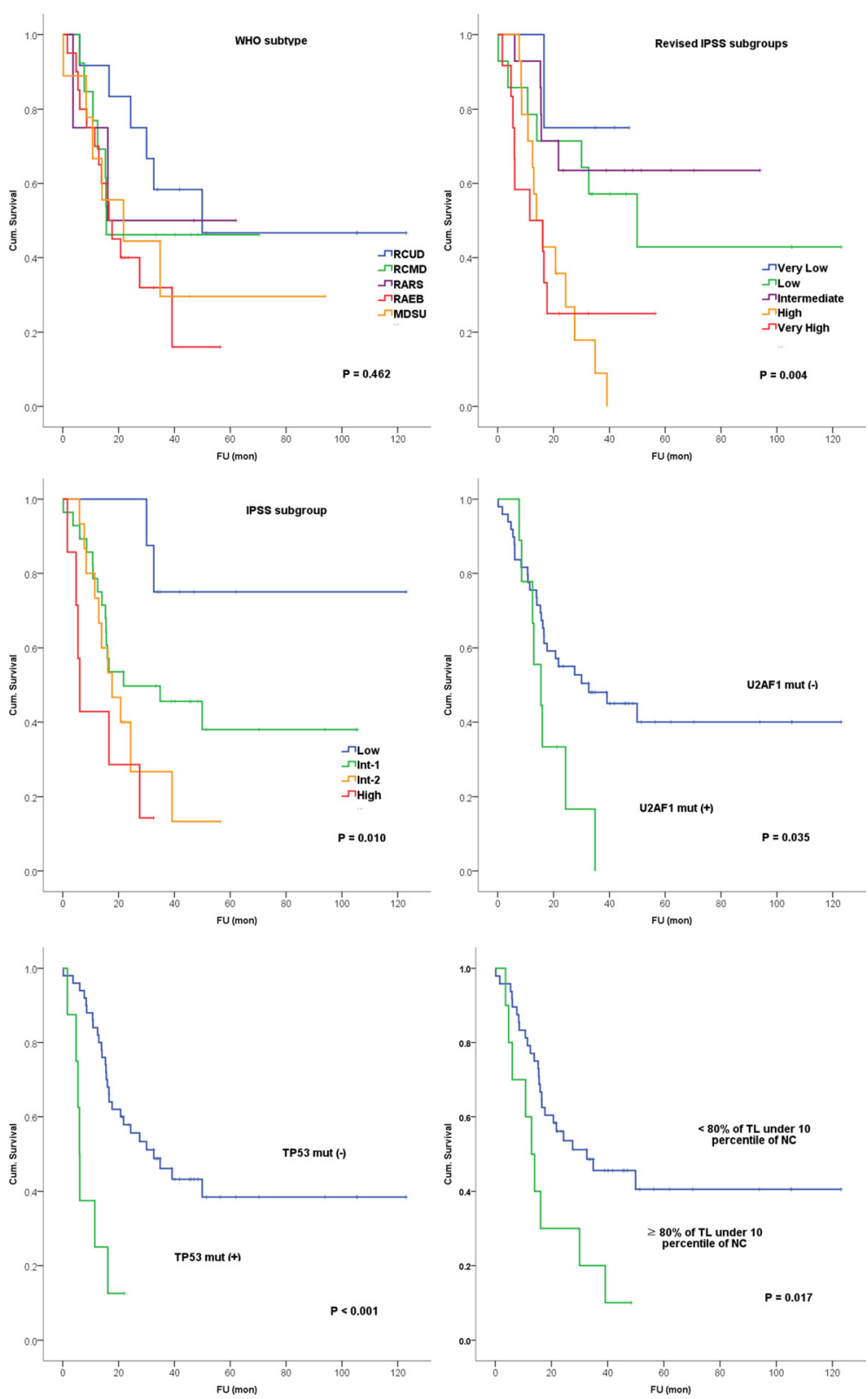

Fig. 5 Kaplan-Meier survival curves according to WHO subtypes, IPSS subgroups, telomere lengths, and TP53 and U2AF1 mutation status 
The OS between those with or without certain gene mutations was compared in genes with mutations that were found in more than $5 \%$ of the patients. We found that patients with TP53 mutation and U2AF1 mutation had a significantly shorter OS compared to those without mutation (median survival 32.6 vs. 6.0 months, $P<0.001$ for TP53 mutation, 32.6 vs. $15.5, P=0.035$ for $U 2 A F 1 \mathrm{mu}-$ tation). We also compared the OS of patients with $\geq 80$ vs. $<80 \%$ of their cells with TL below the lowest 10 th percentile TL of normal controls, and the former was significantly shorter (12.9 vs. 32.6 months, $P=0.017)$. The OS was significantly different in patients with $\geq 80$ vs. $<80 \%$ of their cells with TL below the lowest 10 th percentile TL of the NC even within WHO subtypes and IPSS subgroups $(P=0.038,0.022$, respectively). The presence of other mutations was not related to the OS.

Patients with complex karyotype $(P=0.015)$, del $(7 q) /-7$ by karyotype $(P<0.001)$, abnormalities of $17 \mathrm{p}$ by karyotype $(\mathrm{P}=0.001)$, abnormalities on FISH for chromosome 5 and $7(P=0.014, P<0.001$, respectively), high BM blast count $(\geq 10 \%)(P=0.014)$, and those patients with $\geq 80 \%$ of TL under the 10th percentile of the NC $(P=0.021)$ showed significantly lower survival than those without abnormalities. Univariable and multivariable Cox analyses for overall survival were performed with the clinical parameters, mutational profiles and TL parameters (Table 4). Excluding those parameters with significant correlation and many missing values, multivariable Cox analysis showed that the TP53 mutation, U2AF1 mutation, high BM blast count $(\geq 10 \%)$, and high percentage $(\geq 80 \%)$ of cells with TL less than the 10th percentile of the NC were independent prognostic factors for survival in patients with MDS (Table 4). The Harrell's C-index was $0.649(0.579-0.719)$ for the revised IPSS only and 0.717
(0.654-0.779) for the model including TL, TP53, and $U 2 A F 1$ mutation and blast count showing similar prediction power for survival in our model including TL.

\section{Discussion}

The findings of shortened TL in many MDS patients have led to the studies of telomere dynamics $[9,11,38,39]$ and its relationship with MDS [16]. The shortest TL in an individual, which is considered to be critical in cellular survival and telomere dysfunction [21, 40] was measured by Q-FISH in a single cell level in patients with MDS. We have found out that in addition to the mean TL, the minimum was also shorter in the MDS patients, and the patients showed a narrower range of TL than the NC. In average, $52.2 \%$ of each patient had a TL of lower than the 10th percentile of the NC, suggesting a high burden of cells with critically short TL in the patients with MDS.

Few studies have analyzed the association of mutation status; only that of the telomerase complex-related genes and TL in MDS patients $[17,18]$. Some patients with telomere attrition had TERC or TERT mutations, but only a small number of patients were included in each study [17]. Therefore, we evaluated TL in relation to mutation of myeloid neoplasia-related genes and genes commonly found in hematologic malignancies. Patients without gene mutations had shorter TL than those with mutation (regardless of the gene), but comparing the TL between those and those without each specific gene mutation, the difference was only present for CSF3R gene. In the present study, the population with TL below the 10th percentile TL of the NC was markedly low in patients with CSF3R mutation. It is well known that mutation of CSF3R is accompanied in congenital neutropenia. The congenital neutropenia patients that did not develop leukemia had an incidence of CSF3R mutation in $34 \%$, compared to

Table 4 Univariable and multivariable Cox analysis of the overall survival of 58 MDS patients

\begin{tabular}{|c|c|c|c|c|c|c|}
\hline \multirow[b]{2}{*}{ Risk factors } & \multicolumn{3}{|c|}{ Univariable } & \multicolumn{3}{|c|}{ Multivariable } \\
\hline & $\mathrm{HR}$ & $95 \% \mathrm{Cl}$ & $P$ & $\mathrm{HR}$ & $95 \% \mathrm{Cl}$ & $P$ \\
\hline Revised IPSS & & & 0.010 & & & \\
\hline Very low vs. low & 2.242 & $0.275-18.287$ & 0.451 & & & \\
\hline Very low vs. intermediate & 1.577 & $0.184-13.552$ & 0.678 & & & \\
\hline Very low vs. high & 6.731 & $0.874-51.865$ & 0.067 & & & \\
\hline Very low vs. very high & 6.432 & $0.808-51.197$ & 0.079 & & & \\
\hline Complex CG vs. not & 2.915 & $1.229-6.918$ & 0.015 & & & \\
\hline del(7q)/-7 vs. normal (karyotype) & 4.483 & $1.951-10.302$ & $<0.001$ & & & \\
\hline FISH 5 abnormal vs. normal & 2.994 & $1.248-7.183$ & 0.014 & & & \\
\hline BM blast $\geq 10$ vs. $<10 \%$ & 2.476 & $1.200-5.112$ & 0.014 & 2.623 & $1.237-5.563$ & 0.012 \\
\hline TP53 mutated vs. normal & 4.875 & $2.031-11.703$ & $<0.001$ & 5.257 & $1.341-33.095$ & 0.001 \\
\hline U2AF1 mutated vs. normal & 2.325 & $1.035-5.222$ & 0.041 & 3.544 & $1.485-8.458$ & 0.004 \\
\hline Percentage of cells with low TL compared to NC $\geq 80$ vs. $<80 \%$ & 2.47 & $1.148-5.314$ & 0.021 & 2.248 & $1.023-4.937$ & 0.044 \\
\hline
\end{tabular}


$78 \%$ for patients who showed transformation into acute leukemia [41]. Mutation of CSF3R could confer the proliferative activity to the hematopoietic stem cell, which possibly leads to a transformation to AML. This may be reflected in the TL of patients with CSF3R, having lesser portion of cells with short telomeres and one of our patients showed AML transformation shortly after diagnosis. The mutation site of CSF3R in MDS patients was different from the known site of mutation in congenital neutropenia but two of the patients showed CSF3R mutation reported in myeloid neoplasms such as chronic neutrophilic leukemia or chronic myelomonocytic leukemia [42].

The hypothesis of this study was that TL erosion might be more significant in certain groups of patients with gene mutations that are known be correlated with telomere dysfunction such as SRSF2 [16]. However, as TL is known to be affected by many other factors including age, environment, gender, stress, oxidative stress, and emotion $[20,43,44]$, we were unable to show a correlation of $\mathrm{TL}$ in relation to a single gene mutation except for CSF3R. Contrary to our expectation, we found out that regardless of the gene mutation type, those patients with more than one gene mutation had higher TL than those without mutation. This finding somewhat contrasts with the recently reported result of aplastic anemia patients. Dumitriu et al. reported that telomere attrition and somatic mutations precede monosomy 7 and that the accumulation of short telomere in each chromosome may have a link to the development of aneuploidy in severe aplastic anemia (AA) [45]. This explanation can be applied to MDS evolved from previous AA. However, we infer that telomere attrition in AA exert a different influence on hematopoietic cells in case of MDS. AA has a decreased hematopoietic stem cell pool, but MDS has a dysfunctional hematopoietic stem cell pool, which has already acquired oncogenic mutations. Although the patients with gene mutations had longer TL than those without mutation in this study, the TL was still shorter than those of the NC. Comparisons within MDS groups may be less useful than the comparison of TL within an individual since telomere lengths are affected by many factors $[20,43,44]$ including therapy, which was shown in a study that evaluated TL changes with treatment [46]. Another explanation may be that the mutations found in these patients were in the genes related to splicing machinery, DNA methylation, chromatin modification, or cohesion complexes and they are known to be mutated in MDS patients as driver/oncogenic mutations [14]. Therefore, these oncogenic mutations may have given the MDS cells the characteristics of "cancer" cells, which maintain telomere lengths [47], thus showing longer TL in patients with gene mutation. On the other hand, another explanation may be that these patients without gene mutations may have incorporated a mechanism that further shortens the length of the telomeres in this group. Mutations in other genes not included in this study such as TERC or TERT may give clue to these patients $[17,18]$. Since no other study compared the TL of MDS patients and the presence of genetic mutation commonly found in hematologic malignancies, further studies would help elucidate the relationship between the telomere length and somatic mutations.

In addition, as TL is also regulated by the telomerase activity (TA) [1], we believe that the TA results would give additional information about the telomere dynamics. However, due to the sample limitation, TA could not be measured. TA was measured in MDS in few other studies, and MDS showed normal to low levels of telomerase activity $[9,48]$ despite the short TL. These results suggest the disruption of telomere maintenance in patients with MDS and suggest the maintenance of TL may be to some extent independent of TA incorporating alternative lengthening of telomere mechanisms, as is found in 10-15\% of the tumors [47].

The evaluation of TL with disease progression may be more informative and some reported telomere attrition during progression to acute myeloid leukemia [38, 49] but were shown in a small number of patients, thus requiring further study. In our study, there was no correlation of TL with various clinical subgroups and cytogenetic abnormalities, which is in line with previous studies showing inconsistent association between clinical factors and TL $[9,12]$. Our finding may partly be due to the small number of patients included in our study, which is a major limitation of this study. Since cells with critically shortened telomeres may show repeated fusion bridge cycles, which leads to accumulation of chromosomal abnormalities, the relationship between chromosomal aberrations [50] and TL were also assessed. However, no significant change was found in relation to those with or without chromosomal aberrations. In addition to TP53 mutation, U2AF1 mutation was also suggested to be associated with a poor risk [51,52] and was incorporated in the prognostic model along with the blast count and having high percentage of cells ( $\geq 80 \%$ ) with TL lower than the 10th percentile of the NC. Although the shortest telomere, the minimum TL, which is thought to be critical in the maintenance of cell survival, did not show prognostic significance in our study, we have found that having a high percentage of cells with short TL compared to NC may be of prognostic significance. The short TL was defined by having shorter than the lower 10th percentile value of the NC and this value may already suggest a portion of cells with critically short telomeres. Despite the limitation that Q-FISH measures the relative TL, the comparison made with the $\mathrm{NC}$ allowed us to confirm the shortened 
TL in MDS. Measuring TL in individual cells by Q-FISH also enabled us to identify the portion of cells with the short telomeres, which is not obtainable when measured by Southern blot or QPCR, which can only show the average TL. Moreover, our model including the TL parameter was comparable in the prediction of survival by Harrell's C-index suggesting a probability of incorporation of TL and somatic mutation as a useful marker for prognostication.

\section{Conclusions}

In summary, we evaluated the telomere lengths of MDS patients at a single-cell level by quantitative FISH assessing the shortest TL in each individual along with the average and distribution of TLs. Patients with myelodysplastic syndrome showed short telomere lengths compared to the control with a significant proportion of cells having shorter telomeres than the 10th percentile of the normal control. A proportion of cell population with shorter TL below the 10th percentile of the normal control was significantly larger. We conclude that MDS is characterized by concentrated hematopoietic cells with short telomere, a kind of senescent cells. There were no specific somatic mutation or cytogenetic aberration correlating with short $\mathrm{TL}$, but patients without somatic mutation harbored short telomere than those with somatic mutation. Interestingly, CSF3R mutation correlated with longer telomere length, and proportion of short telomere length was significantly small. TL parameter showed independent prognostic significance and incorporating somatic mutation and TL in addition to known prognostic markers may give additional prediction power for prognosis.

\section{Additional file}

Additional file 1: Supplementary method. Figure S1. Correlation of gene categories and cytogenetic abnormalities. Table S1. Genes included in the target sequencing and its gene categories. Table S2. Telomere lengths according to WHO categories or IPSS subgroups. Table S3. Telomere lengths according to laboratory results. Table $\$ 4$. Telomere lengths according to mutations in gene groups. (DOC 803 kb)

\section{Abbreviations \\ AML, acute myeloid leukemia; BM, bone marrow; FISH, fluorescence in situ hybridization; IPSS, International Prognostic Scoring System; NC, normal control; MDS, myelodysplastic syndrome; OS, overall survival; SD, standard distribution; T/C, telomere to centromere; $\mathrm{TL}$, telomere length; WHO, World Health Organization}

\section{Acknowledgements}

The authors thank the Division of Statistics in Medical Research Collaborating Center at Seoul National University Bundang Hospital for the statistical assistance.

\section{Funding}

This research was supported by the Basic Science Research Program through the National Research Foundation of Korea (NRF) funded by the Ministry of Science, ICT and Future Planning (NRF-2014R1A2A1A10052286) and the Ministry of Education (NRF-2015R1D1A1A02062419) of South Korea. The funding sources played no role in the design; in the collection, analysis, and interpretation of the data; in the writing of the manuscript; and in the decision to submit the manuscript for publication.

\section{Availability of data and materials}

Although all our data is de-identified, we opt not to share the data and materials in public due to further study on this subject. However, we will share the data in request by other researchers if necessary. All of the methods including the software programs or reagents used in this study are on market, which are accessible by other researchers.

\section{Authors' contributions}

SMH, SYK, KOI, SNP, JAK, HSP, SMK, and KK performed the research. JAK, SMH, SYK, and DSL designed the research study. SNP, KOI, KK, SMK, and SMH analyzed the data. SMH, SYK, and DSL wrote the paper. All authors read and approved the final manuscript.

\section{Competing interests}

The authors declare that they have no competing interests.

\section{Consent for publication}

Not applicable.

\section{Ethics approval and consent to participate}

This study was approved by the institutional review board of Seoul National University Hospital (1604-082-754), and the participants gave informed consent.

\section{Author details}

${ }^{1}$ Department of Laboratory Medicine, Seoul National University Bundang Hospital, Seongnam, Republic of Korea. ${ }^{2}$ Department of Laboratory Medicine, Seoul National University College of Medicine, Seoul, Republic of Korea. ${ }^{3}$ Department of Laboratory Medicine, Chungnam National University College of Medicine, Daejeon, Republic of Korea. ${ }^{4}$ Cancer Research Institute, Seoul National University College of Medicine, Seoul, Republic of Korea.

Received: 27 May 2016 Accepted: 11 July 2016

Published online: 28 July 2016

\section{References}

1. Greider CW. Telomere length regulation. Ann Rev Biochem. 1996;65(1):337-65.

2. McClintock B. The behavior in successive nuclear divisions of a chromosome broken at meiosis. Proc Natl Acad Sci U S A. 1939;25(8):405-16.

3. Capper R, Britt-Compton B, Tankimanova M, Rowson J, Letsolo B, Man S, et al. The nature of telomere fusion and a definition of the critical telomere length in human cells. Genes Dev. 2007;21(19):2495-508.

4. Bechter OE, Eisterer W, Pall G, Hilbe W, Kühr T, Thaler J. Telomere length and telomerase activity predict survival in patients with B cell chronic lymphocytic leukemia. Cancer Res. 1998;58(21):4918-22.

5. Wu KD, Orme LM, Shaughnessy J, Jacobson J, Barlogie B, Moore MA. Telomerase and telomere length in multiple myeloma: correlations with disease heterogeneity, cytogenetic status, and overall survival. Blood. 2003;101(12):4982-9.

6. Aalbers AM, Calado RT, Young NS, Zwaan CM, Wu C, Kajigaya S, et al. Telomere length and telomerase complex mutations in pediatric acute myeloid leukemia. Leukemia. 2013;27(8):1786-9.

7. Hofmann WK, Koeffler HP. Myelodysplastic syndrome. Annu Rev Med. 2005;56:1-16.

8. Vardiman J, Arber D, Brunning R, Larson R, Matutes E, Baumann I. WHO classification of tumours of haematopoietic and lymphoid tissues. Lyon: International Agency for Research on Cancer; 2008.

9. Ohyashiki JH, Iwama H, Yahata N, Ando K, Hayashi S, Shay JW, et al. Telomere stability is frequently impaired in high-risk groups of patients with myelodysplastic syndromes. Clin Cancer Res. 1999;5(5):1155-60.

10. Rollison DE, Epling-Burnette P, Park JY, Lee JH, Park H, Jonathan K, et al. Telomere length in myelodysplastic syndromes. Leuk Lymphoma. 2011;52(8):1528-36.

11. Boultwood J, Fidler C, Kusec R, Rack K, Elliott P, Atoyebi O, et al. Telomere length in myelodysplastic syndromes. Am J Hematol. 1997;56(4):266-71. 
12. Lange K, Holm L, Vang Nielsen K, Hahn A, Hofmann W, Kreipe H, et al. Telomere shortening and chromosomal instability in myelodysplastic syndromes. Genes Chromosomes Cancer. 2010;49(3):260-9.

13. Haferlach $T$, Nagata $Y$, Grossmann $V$, Okuno $Y$, Bacher U, Nagae $G$, et al. Landscape of genetic lesions in 944 patients with myelodysplastic syndromes. Leukemia. 2014;28(2):241-7.

14. Papaemmanuil E, Gerstung M, Malcovati L, Tauro S, Gundem G, Van Loo P, et al. Clinical and biological implications of driver mutations in myelodysplastic syndromes. Blood. 2013;122(22):3616-27.

15. Graubert TA, Shen D, Ding L, Okeyo-Owuor T, Lunn CL, Shao J, et al. Recurrent mutations in the U2AF1 splicing factor in myelodysplastic syndromes. Nat Genet. 2012;44(1):53-7.

16. Colla S, Ong DST, Ogoti Y, Marchesini M, Mistry NA, Clise-Dwyer K, et al. Telomere dysfunction drives aberrant hematopoietic differentiation and myelodysplastic syndrome. Cancer Cell. 2015;27(5):644-57.

17. Yamaguchi H. Mutations of the human telomerase RNA gene (TERC) in aplastic anemia and myelodysplastic syndrome. Blood. 2003;102(3):916-8.

18. Kirwan M, Vulliamy T, Marrone A, Walne AJ, Beswick R, Hillmen P, et al. Defining the pathogenic role of telomerase mutations in myelodysplastic syndrome and acute myeloid leukemia. Hum Mut. 2009;30(11):1567-73.

19. Rigolin GM, Porta MD, Bugli AM, Castagnari B, Mauro E, Bragotti LZ, et al. Flow cytometric detection of accelerated telomere shortening in myelodysplastic syndromes: correlations with aetiological and clinical-biological findings*. Eur J Haematol. 2004;73(5):351-8.

20. Sanders $J$, Newman AB. Telomere length in epidemiology: a biomarker of aging, age-related disease, both, or neither? Epidemiol Rev. 2013;35(1):112-31.

21. Hemann MT, Strong MA, Hao LY, Greider CW. The shortest telomere, not average telomere length, is critical for cell viability and chromosome stability. Cell. 2001;107(1):67-77.

22. Perner S, Brüderlein S, Hasel C, Waibel I, Holdenried A, Ciloglu N, et al. Quantifying telomere lengths of human individual chromosome arms by centromere-calibrated fluorescence in situ hybridization and digital imaging. Am J Pathol. 2003;163(5):1751-6.

23. Narath $R$, Lörch T, Greulich-Bode KM, Boukamp P, Ambros PF. Automatic telomere length measurements in interphase nuclei by IQ-FISH. Cytometry A. 2005;68(2):113-20.

24. Lansdorp PM, Verwoerd NP, Van De Rijke FM, Dragowska V, Little MT, Dirks RW, et al. Heterogeneity in telomere length of human chromosomes. Hum Mol Genet. 1996;5(5):685-91.

25. Czepulkowski B, Bhatt B, Rooney D. Basic techniques for the preparation and analysis of chromosomes from bone marrow and leukaemic blood. Human cytogenetics: malignancy and acquired abnormalities. 3rd ed. Oxford: Oxford University Press; 2001. p. 1-26.

26. Shaffer L, Slovak M, Campbell L. ISCN 2009. An international system for human cytogenetics nomenclature. 2009.

27. Shaffer LG, McGowan-Jordan, J, Schmid, M. ISCN 2013: an international system for human cytogenetic nomenclature (2013). Basel: Karger Medical and Scientific Publishers; 2013.

28. Lee DS, Kim SH, Seo EJ, Park CJ, Chi HS, Ko EK, et al. Predominance of trisomy $1 \mathrm{q}$ in myelodysplastic syndromes in Korea: is there an ethnic difference? A 3-year multi-center study. Cancer Genet Cytogenet. 2002;132(2):97-101.

29. Walter M, Shen D, Shao J, Ding L, White B, Kandoth C, et al. Clonal diversity of recurrently mutated genes in myelodysplastic syndromes. Leukemia. 2013;27(6):1275-82.

30. Chapman MA, Lawrence MS, Keats JJ, Cibulskis K, Sougnez C, Schinzel AC, et al. Initial genome sequencing and analysis of multiple myeloma. Nature. 2011;471(7339):467-72.

31. Maxson JE, Gotlib J, Pollyea DA, Fleischman AG, Agarwa A, Eide CA, et al. Oncogenic CSF3R mutations in chronic neutrophilic leukemia and atypical CML. N Engl J Med. 2013;368(19):1781-90.

32. Wang L, Lawrence MS, Wan Y, Stojanov P, Sougnez C, Stevenson K, et al. SF3B1 and other novel cancer genes in chronic lymphocytic leukemia. N Eng J Med. 2011;365(26):2497-506.

33. Landau DA, Carter SL, Stojanov P, McKenna A, Stevenson K, Lawrence MS, et al. Evolution and impact of subclonal mutations in chronic lymphocytic leukemia. Cell. 2013;152(4):714-26.

34. Doménech E, Gómez-López G, Gzlez-Peña D, López M, Herreros B, Menezes J, et al. New mutations in chronic lymphocytic leukemia identified by target enrichment and deep sequencing. Plos One. 2012;7(6):e38158.
35. Tenedini E, Bernardis I, Artusi V, Artuso L, Roncaglia E, Guglielmelli P, et al. Targeted cancer exome sequencing reveals recurrent mutations in myeloproliferative neoplasm. Leukemia. 2014;28:1052-9.

36. Greenberg PL, Tuechler H, Schanz J, Sanz G, Garcia-Manero G, Solé F, et al. Revised international prognostic scoring system for myelodysplastic syndromes. Blood. 2012;120(12):2454-65.

37. Greenberg P, Cox C, LeBeau MM, Fenaux P, Morel P, Sanz G, et al. International scoring system for evaluating prognosis in myelodysplastic syndromes. Blood. 1997:89(6):2079-88.

38. Ohyashik K, Iwama H, Yahata N, Tauchi T, Kawakubo K, Shimamoto T, et al. Telomere dynamics in myelodysplastic syndromes and acute leukemic transformation. Leuk Lymphoma. 2001;42(3):291-9.

39. Sieglová Z, Žilovcová S, Čermák J, Říhová H, Březinová D, Dvořáková R, et al. Dynamics of telomere erosion and its association with genome instability in myelodysplastic syndromes (MDS) and acute myelogenous leukemia arising from MDS: a marker of disease prognosis? Leuk Res. 2004;28(10):1013-21.

40. Abdallah P, Luciano P, Runge KW, Lisby M, Géli V, Gilson E, et al. A two-step model for senescence triggered by a single critically short telomere. Nat Cell Biol. 2009;11(8):988-93.

41. Zeidler C, Germeshausen M, Klein C, Welte K. Clinical implications of ELA2, HAX1- and G-CSF-receptor (CSF3R) mutations in severe congenital neutropenia. Brit J Haematol. 2009;144:459-67.

42. Germeshausen M, Ballmaier M, Welte K. Incidence of CSF3R mutations in severe congenital neutropenia and relevance for leukemogenesis: results of a long-term survey. Blood. 2007;109(1):93-9.

43. Gardner M, Bann D, Wiley L, Cooper R, Hardy R, Nitsch D, et al. Gender and telomere length: systematic review and meta-analysis. Exp Gerontol. 2014;51:15-27.

44. Puterman E, Lin J, Blackburn E, O'Donovan A, Adler N, Epe E. The power of exercise: buffering the effect of chronic stress on telomere length. PLoS One. 2010;5(5):e10837

45. Dumitriu B, Feng X, Townsley DM, Ueda Y, Yoshizato T, Calado RT, et al. Telomere attrition and candidate gene mutations preceding monosomy 7 in aplastic anemia. Blood. 2015;125(4):706-9.

46. Beier F, Masouleh BK, Buesche G, Ferreira MSV, Schneider RK, Ziegler P, et al. Telomere dynamics in patients with del (5q) MDS before and under treatment with lenalidomide. Leuk Res. 2015;39(11):1292-8.

47. Jafri MA, Ansari SA, Alqahtani MH, Shay JW. Roles of telomeres and telomerase in cancer, and advances in telomerase-targeted therapies. Genome Med. 2016;8(1):69.

48. Kim M, Oh B, Kim TY, Yoon SS, Kim SY, Hwang SM, Lee DS. Elevated telomerase activity in essential thrombocythemia with extreme thrombocytosis. Clin Biochem. 2014;47(6):389-92.

49. Göhring G, Lange K, Hofmann W, Nielsen K, Hellström-Lindberg E, Roy L, et al. Telomere shortening, clonal evolution and disease progression in myelodysplastic syndrome patients with $5 \mathrm{q}$ deletion treated with lenalidomide. Leukemia. 2012;26(2):356-8.

50. Artandi SE, Chang S, Lee S-L, Alson S, Gottlieb GJ, Chin L, et al. Telomere dysfunction promotes non-reciprocal translocations and epithelial cancers in mice. Nature. 2000:406(6796):641-5.

51. Wu SJ, Tang JL, Lin CT, Kuo YY, Li LY, Tseng MH, et al. Clinical implications of U2AF1 mutation in patients with myelodysplastic syndrome and its stability during disease progression. Am J Hematol. 2013;88(11):E277-82.

52. Bejar R, Stevenson KE, Caughey RA, Abdel-Wahab O, Steensma DP, Galili N, et al. Validation of a prognostic model and the impact of mutations in patients with lower-risk myelodysplastic syndromes. J Clin Oncol. 2012;30:3376-82.

\section{Submit your next manuscript to BioMed Central and we will help you at every step:}

- We accept pre-submission inquiries

- Our selector tool helps you to find the most relevant journal

- We provide round the clock customer support

- Convenient online submission

- Thorough peer review

- Inclusion in PubMed and all major indexing services

- Maximum visibility for your research

Submit your manuscript at www.biomedcentral.com/submit 